\title{
Research Article \\ Controllable and Observable Polynomial Description for 2D Noncausal Systems
}

\author{
M. S. Boudellioua \\ Department of Mathematics and Statistics, Sultan Qaboos University, P.O. Box 36, Al-Khodh 123, Oman
}

Received 26 February 2007; Accepted 19 May 2007

Recommended by Tongwen Chen

Two-dimensional state-space systems arise in applications such as image processing, iterative circuits, seismic data processing, or more generally systems described by partial differential equations. In this paper, a new direct method is presented for the polynomial realization of a class of noncausal $2 \mathrm{D}$ transfer functions. It is shown that the resulting realization is both controllable and observable.

Copyright (C) 2007 M. S. Boudellioua. This is an open access article distributed under the Creative Commons Attribution License, which permits unrestricted use, distribution, and reproduction in any medium, provided the original work is properly cited.

\section{INTRODUCTION}

A $2 \mathrm{D}$ system is a system in which information propagates in two independent directions. Multidimensional (nD) systems have found many applications in areas such as image and video processing, geophysical exploration, linear multipass processes, iterative learning control systems, lumped and distributed networks [1]. In his pioneering work, Rosenbrock [2] has used polynomial matrices in a single variable to represent systems described by ordinary differential/difference equations. The success of his approach is mainly due to the computational aspects of the division ring involved. The polynomial matrix approach has been extended to the $2 \mathrm{D}$ case by a number of authors such as Bose [1], Frost and Boudellioua [3, 4], Johnson [5], Pugh et al. [6]. In the case of a $2 \mathrm{D}$ system, the resulting matrix is a two-variable polynomial matrix. Unfortunately, the polynomial ring in two variables is not an Euclidean division ring which makes extensions from $1 \mathrm{D}$ to $2 \mathrm{D}$ in most situations not possible.

\section{CAUSAL 2D SYSTEMS}

State-space models play an important role in the theory of 1D finite-dimensional linear systems. Several authors, for example, Attasi [7], Fornasini and Marchesini [8], and Roesser [9] have proposed different state-space models for 2D discrete systems or systems described by partial differential equations and have suggested some extensions of the usual $1 \mathrm{D}$ notions to the $2 \mathrm{D}$ case. However, it has been shown by
Kung et al. [10] and other authors that Roesser's model is the most satisfactory and the most general since the other models can be embedded in it. Roesser's model is one in which the local state is divided into a horizontal state and a vertical state which are propagated, respectively, horizontally and vertically by first-order difference equations.

The model has the form

$$
\begin{aligned}
x^{h}(i+1, j) & =A_{1} x^{h}(i, j)+A_{2} x^{v}(i, j)+B_{1} u(i, j), \\
x^{v}(i, j+1) & =A_{3} x^{h}(i, j)+A_{4} x^{v}(i, j)+B_{2} u(i, j), \\
y(i, j) & =C_{1} x^{h}(i, j)+C_{2} x^{v}(i, j),
\end{aligned}
$$

where $x^{h}(i, j)$ is the horizontal state vector, $x^{v}(i, j)$ is the vertical state vector, $u(i, j)$ is the input vector, $y(i, j)$ is the output vector, and $A_{1}, A_{2}, A_{3}, A_{4}, B_{1}, B_{2}, C_{1}, C_{2}$ are real constant matrices of appropriate dimensions.

System (1) can be written in the polynomial form

$$
\left[\begin{array}{ll|l}
s I_{n}-A_{1} & -A_{2} & B_{1} \\
-A_{3} & z I_{m}-A_{4} & B_{2} \\
\hline-C_{1} & -C_{2} & 0
\end{array}\right]\left[\begin{array}{c}
x^{h}(i, j) \\
x^{v}(i, j) \\
\hline-u(i, j)
\end{array}\right]=\left[\begin{array}{c}
0 \\
0 \\
\hline-y(i, j)
\end{array}\right],
$$

where $s$ denotes an advance shift operator in the horizontal direction and $z$ denotes an advance shift operator in the vertical direction. The polynomial matrix in $s$ and $z$,

$$
P(s, z)=\left[\begin{array}{cc|c}
s I_{n}-A_{1} & -A_{2} & B_{1} \\
-A_{3} & z I_{m}-A_{4} & B_{2} \\
\hline-C_{1} & -C_{2} & 0
\end{array}\right]
$$


is called a polynomial matrix description (PMD) associated with system (1). The order of $P(s, z)$ is given by $n+m$ and its rational transfer function is

$$
G(s, z)=\left[\begin{array}{ll}
C_{1} & C_{2}
\end{array}\right]\left[\begin{array}{cc}
s I_{n}-A_{1} & -A_{2} \\
-A_{3} & z I_{m}-A_{4}
\end{array}\right]^{-1}\left[\begin{array}{l}
B_{1} \\
B_{2}
\end{array}\right] .
$$

Given the transfer function $G(s, z)$, the associated PMD, $P(s, z)$ that gives rise to $G(s, z)$ is called a realization of $G(s, z)$.

Using the definitions given by Zerz [11], system (1) is said to be strongly controllable if the matrix

$$
\mathcal{C}(s, z)=\left[\begin{array}{cc|c}
s I_{n}-A_{1} & -A_{2} & B_{1} \\
-A_{3} & z I_{m}-A_{4} & B_{2}
\end{array}\right]
$$

has full rank for all $(s, z) \in \mathbb{C}^{2}$ and canonical if furthermore it is observable, that is, the matrix

$$
\mathcal{O}(s, z)=\left[\begin{array}{cc}
s I_{n}-A_{1} & -A_{2} \\
-A_{3} & z I_{m}-A_{4} \\
\hline-C_{1} & -C_{2}
\end{array}\right]
$$

has no nontrivial factors in $\mathbb{R}[s, z]$, that is, any factorization $\mathcal{O}(s, z)=\overline{\mathcal{O}}(s, z) F(s, z)$, where $F(s, z)$ is square, implies that $F(s, z)$ is unimodular.

Another term used in the context of PMDs of the form (3) is that the PMD is said to be separable if the determinant

$$
\left|\begin{array}{cc}
s I_{n}-A_{1} & -A_{2} \\
-A_{3} & z I_{m}-A_{4}
\end{array}\right|
$$

can be written as a product of two polynomials, one in $s$, the other in $z$.

\section{NONCAUSAL 2D SYSTEMS}

One of the limitations of the model given in (1) is that it can only be used to describe causal 2D transfer functions. In other words, it is suitable only for the representation of northeast quarter plane 2D systems. Several authors have suggested a generalized state-space description for $2 \mathrm{D}$ systems. Zak [12] suggested a generalized model based on Roesser's model while Kaczorek [13, 14] proposed a model based on that of Fornasini and Marchesini. Noncausal 2D systems have been studied more recently by a number of authors such as Galkowski [15], Kaczorek [16], Zou and Campbell [17], and Xu et al. [18].

In the following, we will be concerned with PMDs of the type given by Zak [12], that is,

$$
P(s, z)=\left[\begin{array}{ll|l}
s E_{1}-A_{1} & -A_{2} & B_{1} \\
-A_{3} & z E_{2}-A_{4} & B_{2} \\
\hline-C_{1} & -C_{2} & 0
\end{array}\right],
$$

where $A_{1}, A_{2}, A_{3}, A_{4}$ are, respectively, $n \times n, n \times m, m \times n$, $m \times m$ real constant matrices, $E_{1}, E_{2}$ are, respectively, $n \times n$ and $m \times m$ real constant matrices which may be singular, and $B_{1}, B_{2}, C_{1}, C_{2}$ are, respectively, $n \times l, m \times l, p \times n, p \times m$ real constant matrices.
The transfer function corresponding to the PMD (8) is the $p \times l$ rational matrix given by

$$
G(s, z)=\left[\begin{array}{ll}
C_{1} & C_{2}
\end{array}\right]\left[\begin{array}{cc}
s E_{1}-A_{1} & -A_{2} \\
-A_{3} & z E_{2}-A_{4}
\end{array}\right]^{-1}\left[\begin{array}{l}
B_{1} \\
B_{2}
\end{array}\right] .
$$

PMDs of the form (8) can be regarded as extensions of the PMDs over $\mathbb{R}[s]$ of the form

$$
P(s)=\left[\begin{array}{c|c}
s E-A & B \\
\hline-C & 0
\end{array}\right],
$$

which are often encountered in the study of the so-called generalized or descriptor state-space systems developed by Verghese et al. [19]. PMDs of the type (8) have been found to be useful by Zak [12] in the study of systems described by high-order partial differential equations.

One way to establish a connection between PMDs of the form (8) is via Rosenbrock's strict-system equivalence (SSE) defined by the following.

Definition 1. Two PMDs $P(s, z)$ and $Q(s, z)$ of the type (8) and having the same size are said to be SSE if they are related by a transformation of the form

$$
\begin{gathered}
{\left[\begin{array}{c|c}
M(s, z) & 0 \\
\hline X(s, z) & I_{p}
\end{array}\right] \underbrace{\left[\begin{array}{cc|c}
s E_{1}-A_{1} & -A_{2} & B_{1} \\
-A_{3} & z E_{2}-A_{4} & B_{2} \\
\hline-C_{1} & -C_{2} & 0
\end{array}\right]}_{P(s, z)}} \\
=\underbrace{\left[\begin{array}{cc|c}
s F_{1}-L_{1} & -L_{2} & R_{1} \\
-L_{3} & z F_{2}-L_{4} & R_{2} \\
\hline-S_{1} & -S_{2} & 0
\end{array}\right]}_{Q(s, z)}\left[\begin{array}{c|c}
N(s, z) & Y(s, z) \\
\hline 0 & I_{l}
\end{array}\right],
\end{gathered}
$$

where $M(s, z), N(s, z)$ are $(n+m) \times(n+m)$ unimodular polynomial matrices and $X(s, z), Y(s, z)$ are polynomial matrices of appropriate dimensions.

The transformation of SSE given in (11) preserves the transfer function and other system structural properties such as controllability and observability. However, the main disadvantage of using SSE in the context of PMDs of the type (8) is that it does not preserve the state space form (8). So in the case when two PMDs $P(s, z)$ and $Q(s, z)$ are in statespace form (8) and have the same dimensions, we introduce the following notion of restricted-system equivalence (RSE).

Definition 2. Two PMDs $P(s, z)$ and $Q(s, z)$ of the type (8) are said to be RSE if they are related by a transformation of the form

$$
\begin{gathered}
{\left[\begin{array}{ll|l}
M_{1} & 0 & 0 \\
0 & M_{2} & 0 \\
\hline 0 & 0 & I_{p}
\end{array}\right] \underbrace{\left[\begin{array}{ll|l}
s E_{1}-A_{1} & -A_{2} & B_{1} \\
-A_{3} & z E_{2}-A_{4} & B_{2} \\
\hline-C_{1} & -C_{2} & 0
\end{array}\right]}_{P(s, z)}} \\
=\underbrace{\left[\begin{array}{ll|l}
s F_{1}-L_{1} & -L_{2} & R_{1} \\
-L_{3} & z F_{2}-L_{4} & R_{2} \\
\hline-S_{1} & -S_{2} & 0
\end{array}\right]}_{Q(s, z)}\left[\begin{array}{ll|l}
N_{1} & 0 & 0 \\
0 & N_{2} & 0 \\
\hline 0 & 0 & I_{l}
\end{array}\right],
\end{gathered}
$$


where $M_{1}, M_{2}, N_{1}, N_{2}$ are nonsingular real constant matrices of appropriate dimensions.

The transformation of RSE in (12) is clearly a special case of the general SSE given in (11), and therefore preserves the transfer function matrix (9) and the controllability and observability properties of the original system. Note that when $E_{1}$ and $E_{2}$ are nonsingular, the PMD $P(s, z)$ is RSE to a PMD of the type (3), that is, with $F_{1}=I_{n}$ and $F_{2}=I_{m}$.

In the following, we will be concerned with PMDs having both $E_{1}$ and $E_{2}$ singular. First, we present a result for reducing a PMD of the type (8) to a canonical form using an RSE transformation.

Theorem 1. Let $P(s, z)$ be an $(n+m+p) \times(n+m+l) P M D$ in state-space form (8) with $\left|s E_{1}-A_{1}\right| \not \equiv 0$ and $\left|z E_{2}-A_{4}\right| \not \equiv 0$. Then $P(s, z)$ is RSE to a PMD of the form

$$
\bar{P}(s, z)=\left[\begin{array}{cccc|c}
s I_{r}-\bar{A}_{1} & 0 & -\bar{A}_{21} & -\bar{A}_{22} & B_{1 s} \\
0 & I_{n-r}-s J_{1} & -\bar{A}_{23} & -\bar{A}_{24} & B_{1 f} \\
-\bar{A}_{31} & -\bar{A}_{32} & z I_{t}-\bar{A}_{4} & 0 & B_{2 s} \\
-\bar{A}_{33} & -\bar{A}_{34} & 0 & I_{m-t}-z J_{2} & B_{2 f} \\
\hline-C_{1 s} & -C_{1 f} & -C_{2 s} & -C_{2 f} & 0
\end{array}\right],
$$

where $\bar{A}_{1}, \bar{A}_{4}$ are, respectively, $r \times r, t \times t$ matrices in first natural form $\left(r=\operatorname{deg}\left(\left|s E_{1}-A_{1}\right|\right), t=\operatorname{deg}\left(\left|z E_{2}-A_{4}\right|\right)\right)$ and $J_{1}$ and $J_{2}$ are in Jordan form.

Proof. This result follows from the theory of regular matrix pencils given by Gantmacher [20].

Example 1. Consider the PMD

$$
P(s, z)=\left[\begin{array}{ccccc|c}
s-1 & s-1 & 0 & -2 & -2 & 1 \\
-1 & s & s & -3 & 2 & 1 \\
-1 & 0 & 1 & 2 & -1 & 1 \\
-2 & -3 & 1 & z-2 & z-2 & 0 \\
-2 & -6 & 1 & z-2 & z-1 & 1 \\
\hline-2 & -1 & 0 & -3 & -1 & 0
\end{array}\right]
$$

Here, $n=3, m=2$, and $l=p=1$. The matrices in (8) are given by

$$
\begin{array}{cc}
E_{1}=\left[\begin{array}{lll}
1 & 1 & 0 \\
0 & 1 & 1 \\
0 & 0 & 0
\end{array}\right], \quad A_{1}=\left[\begin{array}{ccc}
1 & 1 & 0 \\
1 & 0 & 0 \\
1 & 0 & -1
\end{array}\right], & A_{2}=\left[\begin{array}{cc}
2 & 2 \\
3 & -2 \\
-2 & 1
\end{array}\right], \\
A_{3}=\left[\begin{array}{lll}
2 & 3 & -1 \\
2 & 6 & -1
\end{array}\right], \quad E_{2}=\left[\begin{array}{ll}
1 & 1 \\
1 & 1
\end{array}\right], & A_{4}=\left[\begin{array}{ll}
2 & 2 \\
2 & 1
\end{array}\right] .
\end{array}
$$

Then, clearly $\left|s E_{1}-A_{1}\right|=s-1 \not \equiv 0$ and $\left|z E_{2}-A_{4}\right|=z-2 \not \equiv 0$. It follows that the RSE transformation of the type (12) with

$$
\begin{array}{rlrl}
M_{1} & =\left[\begin{array}{ccc}
1 & 0 & 0 \\
-1 & 1 & 0 \\
0 & 0 & -1
\end{array}\right], & M_{2}=\left[\begin{array}{cc}
1 & 0 \\
-1 & 1
\end{array}\right], \\
N_{1}=\left[\begin{array}{ccc}
1 & -1 & 0 \\
0 & 1 & 0 \\
1 & -1 & -1
\end{array}\right], & N_{2}=\left[\begin{array}{cc}
1 & -1 \\
0 & 1
\end{array}\right]
\end{array}
$$

reduces $P(s, z)$ to the PMD in the form (13):

$$
\bar{P}(s, z)=\left[\begin{array}{ccccc|c}
s-1 & 0 & 0 & -2 & 0 & 1 \\
0 & 1 & -s & -1 & 5 & 0 \\
0 & 0 & 1 & -2 & 3 & -1 \\
-1 & -2 & -1 & z-2 & 0 & 0 \\
0 & -3 & 0 & 0 & 1 & 1 \\
\hline-2 & 1 & 0 & -3 & 2 & 0
\end{array}\right]
$$

\section{CANONICAL PMD OF 2D NONCAUSAL SYSTEMS}

The problem of finding a realization which is minimal in some sense is important in multidimensional systems theory. Zak [12] used a similar approach to that of Eising [21] for $2 \mathrm{D}$ causal systems to obtain a two-level algorithm for the realization of a class of noncausal 2D transfer functions. However, the method given by Zak does not result in a realization which is necessarily minimal. More recently, progress has been made for special classes of systems, see for example Galkowski [15] and Kaczorek [16]. In the following, we give a new direct method for a canonical realization of another class of SISO noncausal 2D transfer functions. Furthermore, the realization obtained is both controllable and observable.

Algorithm 1. Consider the SISO noncausal 2D transfer function:

$$
g(s, z)=\frac{\tilde{n}(s, z)}{d(s, z)}
$$

where

$$
\begin{aligned}
\tilde{n}(s, z) & =r(s, z) d(s, z)+n(s), \\
n(s) & =e_{n} s^{n}+e_{n-1} s^{n-1}+\cdots+e_{0}, \\
d(s, z) & =k_{0}(s) z^{m}+k_{1}(s) z^{m-1}+\cdots+k_{m}(s),
\end{aligned}
$$

$k_{0}(s)$ is monic and has degree equal to $n$, and $k_{j}(s), j=$ $1,2, \ldots, m$ have degree less or equal to $n$, and $\tilde{n}(s, z)$ and $d(s, z)$ are factor coprime. Furthermore,

$$
r(s, z)=r_{q+1}(s) z^{q}+r_{q}(s) z^{q-1}+\cdots+r_{1}(s),
$$

where

$$
r_{i}(s)=\sum_{j=1}^{l+1} w_{i j} s^{l-j+1}, \quad i=1,2, \ldots, q+1,
$$

where $l=\operatorname{deg}_{s} r(s, z)$. Then $g(s, z)$ can be decomposed as

$$
g(s, z)=g_{0}(s, z)+r(s, z)
$$

where

$$
g_{0}(s, z)=\frac{n(s)}{d(s, z)} .
$$

It follows using the algorithm given by Frost and Boudellioua [4] that $g_{0}(s, z)$ can be realized by a PMD of the form

$$
P_{0}(s, z)=\left[\begin{array}{cc|c}
s I_{n}-F_{1} & -\bar{A}_{2} & 0 \\
-\bar{A}_{3} & z I_{m}-F_{4} & E_{m} \\
\hline-C_{1} & -C_{2} & 0
\end{array}\right],
$$


where $F_{1}$ and $F_{4}$ are $n \times n$ and $m \times m$ companion matrices and $\bar{A}_{2}=\left[\begin{array}{ll}E_{n} & 0\end{array}\right]$. The elements of $F_{1}, F_{4}$, and $\bar{A}_{3}$ are uniquely determined from the characteristic polynomial

$$
\left|\begin{array}{cc}
s I_{n}-F_{1} & -\bar{A}_{2} \\
-\bar{A}_{3} & z I_{m}-F_{4}
\end{array}\right| .
$$

Furthermore, if $d(s, z)$ is separable, then $\bar{A}_{3}=0$.

On the other hand, it can be easily verified that the PMD

$$
P_{r}(s, z)=\left[\begin{array}{cc|c}
I_{l+1}-s J_{1} & 0 & E_{l+1} \\
-\bar{W} & I_{q+1}-z J_{2} & 0 \\
\hline 0 & -E_{1}^{T} &
\end{array}\right]
$$

is a realization of $r(s, z)$, where

$$
\bar{W}=\left[w_{i j}\right], \quad i=1,2, \ldots, q+1, j=1,2, \ldots, l+1,
$$

and $E_{l+1}$ is the $(l+1)$ th column of the identity matrix $I_{l+1}$.

Therefore, the PMD

$$
P_{1}(s, z)=\left[\begin{array}{cccc|c}
s I_{n}-F_{1} & -\bar{A}_{2} & 0 & 0 & 0 \\
-\bar{A}_{3} & z I_{m}-F_{4} & 0 & 0 & E_{m} \\
0 & 0 & I_{l+1}-s J_{1} & 0 & E_{l+1} \\
0 & 0 & -\bar{W} & I_{q+1}-z J_{2} & 0 \\
\hline-C_{1} & -C_{2} & 0 & -E_{1}^{T} & 0
\end{array}\right],
$$

where $F_{1}, F_{4}, \bar{A}_{2}, \bar{A}_{3}, \bar{C}_{1}$, and $\bar{C}_{2}$ are the matrices which appear in the realization of $g_{0}(s, z)$, is a realization of $g(s, z)$.

$P_{1}(s, z)$ in (28) can be rearranged by elementary row and column operations to yield a PMD in the form (8)

$$
\bar{P}(s, z)\left[\begin{array}{cccc|c}
s I_{n}-F_{1} & 0 & -\bar{A}_{2} & 0 & 0 \\
0 & I_{l+1}-s J_{1} & 0 & 0 & E_{l+1} \\
-\bar{A}_{3} & 0 & z I_{m}-F_{4} & 0 & E_{m} \\
0 & -\bar{W} & 0 & I_{q+1}-z J_{2} & 0 \\
\hline-C_{1} & 0 & -C_{2} & -E_{1}^{T} & 0
\end{array}\right] .
$$

Now it remains to show that the $\bar{P}(s, z)$ in (29) is canonical. The strong controllability of $\bar{P}(s, z)$ follows from the fact that $\bar{P}(s, z)$ can be reduced by SSE, in this case by elementary row and column operations, to the form

$$
S(s, z)=\left[\begin{array}{cc|c}
I_{n+m+l+q+1} & 0 & 0 \\
0 & d(s, z) & 1 \\
\hline 0 & -\widetilde{n}(s, z) & 0
\end{array}\right],
$$

where the PMD, S( $s, z)$, clearly satisfies the condition in (5) and since the polynomials $\tilde{n}(s, z)$ and $d(s, z)$ are factor coprime, it follows that $S(s, z)$ and therefore $\bar{P}(s, z)$ are canonical.

Example 2. Consider the transfer function

$$
\begin{gathered}
g(s, z)=\frac{1}{d(s, z)}\left[\left(s^{3}+2 s^{2}+2 s+1\right) z^{3}+\left(s^{3}+3 s^{2}+5 s+1\right) z^{2}\right. \\
\left.+2\left(s^{3}+2 s^{2}\right) z+2 s^{3}-2 s^{2}-3\right]
\end{gathered}
$$

where

$$
d(s, z)=\left(s^{2}+s+1\right) z^{2}+(3 s+2) z+2 s^{2}-s+2 .
$$

Then

$$
g(s, z)=g_{0}(s, z)+r(s, z)
$$

where

$$
\begin{aligned}
g_{0}(s, z) & =\frac{s^{2}-3 s-1}{\left(s^{2}+s+1\right) z^{2}+(3 s+2) z+2 s^{2}-s+2}, r(s, z) \\
& =(s+1) z+s-1 .
\end{aligned}
$$

Here $n=m=2$ and $l=q=1 \cdot g_{0}(s, z)$ can be realized by the PMD

$$
P_{0}(s, z)=\left[\begin{array}{cccc|c}
s & -1 & 0 & 0 & 0 \\
1 & s+1 & -1 & 0 & 0 \\
2 & 3 & z & -1 & 0 \\
0 & -3 & 2 & z & 1 \\
\hline 2 & 4 & -1 & 0 & 0
\end{array}\right]
$$

It remains to find a realization for $r(s, z)$. Here $r_{1}(s)=s-1$, $r_{2}(s)=s+1$. Therefore,

$$
\bar{W}=\left[\begin{array}{cc}
1 & -1 \\
1 & 1
\end{array}\right]
$$

so that the PMD

$$
P_{r}(s, z)=\left[\begin{array}{cccc|c}
1 & -s & 0 & 0 & 0 \\
0 & 1 & 0 & 0 & 1 \\
-1 & 1 & 1 & -z & 0 \\
-1 & -1 & 0 & 1 & 0 \\
\hline 0 & 0 & -1 & 0 & 0
\end{array}\right]
$$

is a realization of $r(s, z)$.

Finally, it can be easily verified that the PMD

$$
\bar{P}(s, z)=\left[\begin{array}{cccccccc|c}
s & -1 & 0 & 0 & 0 & 0 & 0 & 0 & 0 \\
1 & s+2 & 0 & 0 & -1 & 0 & 0 & 0 & 0 \\
0 & 0 & 1 & -s & 0 & 0 & 0 & 0 & 0 \\
0 & 0 & 0 & 1 & 0 & 0 & 0 & 0 & 1 \\
2 & 3 & 0 & 0 & z & -1 & 0 & 0 & 0 \\
0 & -3 & 0 & 0 & 2 & z & 0 & 0 & 1 \\
0 & 0 & -1 & 1 & 0 & 0 & 1 & -z & 0 \\
0 & 0 & -1 & -1 & 0 & 0 & 0 & 1 & 0 \\
\hline 2 & 4 & 0 & 0 & -1 & 0 & -1 & 0 & 0
\end{array}\right]
$$

is canonical and gives rise to $g(s, z)$.

\section{CONCLUSIONS}

A new method for the realization of a class of noncausal 2D transfer functions has been presented. The resulting polynomial description is canonical in the sense that it is both strongly controllable and observable. 


\section{ACKNOWLEDGMENTS}

The author wishes to express his thanks to Sultan Qaboos University for its support in carrying out this research work and the anonymous reviewers for useful comments.

\section{REFERENCES}

[1] N. K. Bose, Applied Multidimensional Systems Theory, Van Nostrand Reinhold, New York, NY, USA, 1982.

[2] H. H. Rosenbrock, State Space and Multivariable Theory, Nelson-Wiley, New York, NY, USA, 1970.

[3] M. G. Frost and M. S. Boudellioua, "Some further results concerning matrices with elements in a polynomial ring," International Journal of Control, vol. 43, no. 5, pp. 1543-1555, 1986.

[4] M. G. Frost and M. S. Boudellioua, "Further observations concerning the strict system equivalence of polynomial system matrices over $\mathbb{R}[s, z]$," International Journal of Control, vol. 49, no. 1, pp. 1-14, 1989.

[5] D. S. Johnson, Coprimeness in multidimensional system theory and symbolic computation, Ph.D. thesis, Loughborough University of Technology, Leics, UK, 1993.

[6] A. C. Pugh, S. J. Mcinerney, M. S. Bouoellioua, D. S. Johnson, and G. E. Hayton, "A transformation for 2-D linear systems and a generalization of a theorem of Rosenbrock," International Journal of Control, vol. 71, no. 3, pp. 491-503, 1998.

[7] S. Attasi, "Systemes lineaires a deux indices," Tech. Rep. 31, IRIA, Le Chesnay, France, 1973.

[8] E. Fornasini and G. Marchesini, "State space realization theory of two dimensional filters," IEEE Transactions on Automatic Control, vol. 21, no. 4, pp. 484-492, 1976.

[9] R. P. Roesser, "A discrete state-space model for linear image processing," IEEE Transactions on Automatic Control, vol. 20, no. 1, pp. 1-75, 1975.

[10] S.-Y. Kung, B. C. Levy, M. Morf, and T. Kailath, "New results in 2-D systems theory-part II: 2-D state space models, realization and the notions of controllability, observability and minimality," Proceedings of the IEEE, vol. 65, no. 6, pp. 945961, 1977.

[11] E. Zerz, Topics in Multidimensional Linear Systems Theory, Springer, London, UK, 2000.

[12] S. H. Zak, "On state-space models for systems described by partial differential equations," in Proceedings of the 33rd IEEE Conference on Decision and Control (CDC '84), pp. 571-576, Las Vegas, Nev, USA, December 1984.

[13] T. Kaczorek, Two-Dimensional Linear Systems, Springer, London, UK, 1985.

[14] T. Kaczorek, "Singular models of 2-D systems," in Proceedings of the 12th World Congress on Scientific Computation, Paris, France, July 1988.

[15] K. Galkowski, State-Space Realizations of Linear 2-D Systems with Extensions to the General $n D(n>2)$ Case, Springer, London, UK, 2001.

[16] T. Kaczorek, Polynomial and Rational Matrices: Applications in Dynamical Systems Theory, Springer, London, UK, 2006.

[17] Y. Zou and S. L. Campbell, "The jump behavior and stability analysis for 2-D singular systems," Multidimensional Systems and Signal Processing, vol. 11, no. 4, pp. 321-338, 2000.

[18] H. Xu, L. Xie, S. Xu, and Y. Zou, "Positive real control for uncertain 2-D singular Roesser models," International Journal of Control, Automation and Systems, vol. 3, no. 2, pp. 195-201, 2005.
[19] G. C. Verghese, B. C. Levy, and T. Kailath, "A generalized statespace for singular systems," IEEE Transactions on Automatic Control, vol. 26, no. 4, pp. 811-831, 1981.

[20] F. R. Gantmacher, The Theory of Matrices, Chelsea, London, UK, 1971.

[21] R. Eising, 2-D Systems, an Algebraic Approach, Mathematical Centre Tracts, Amsterdam, The Netherland, 1980. 

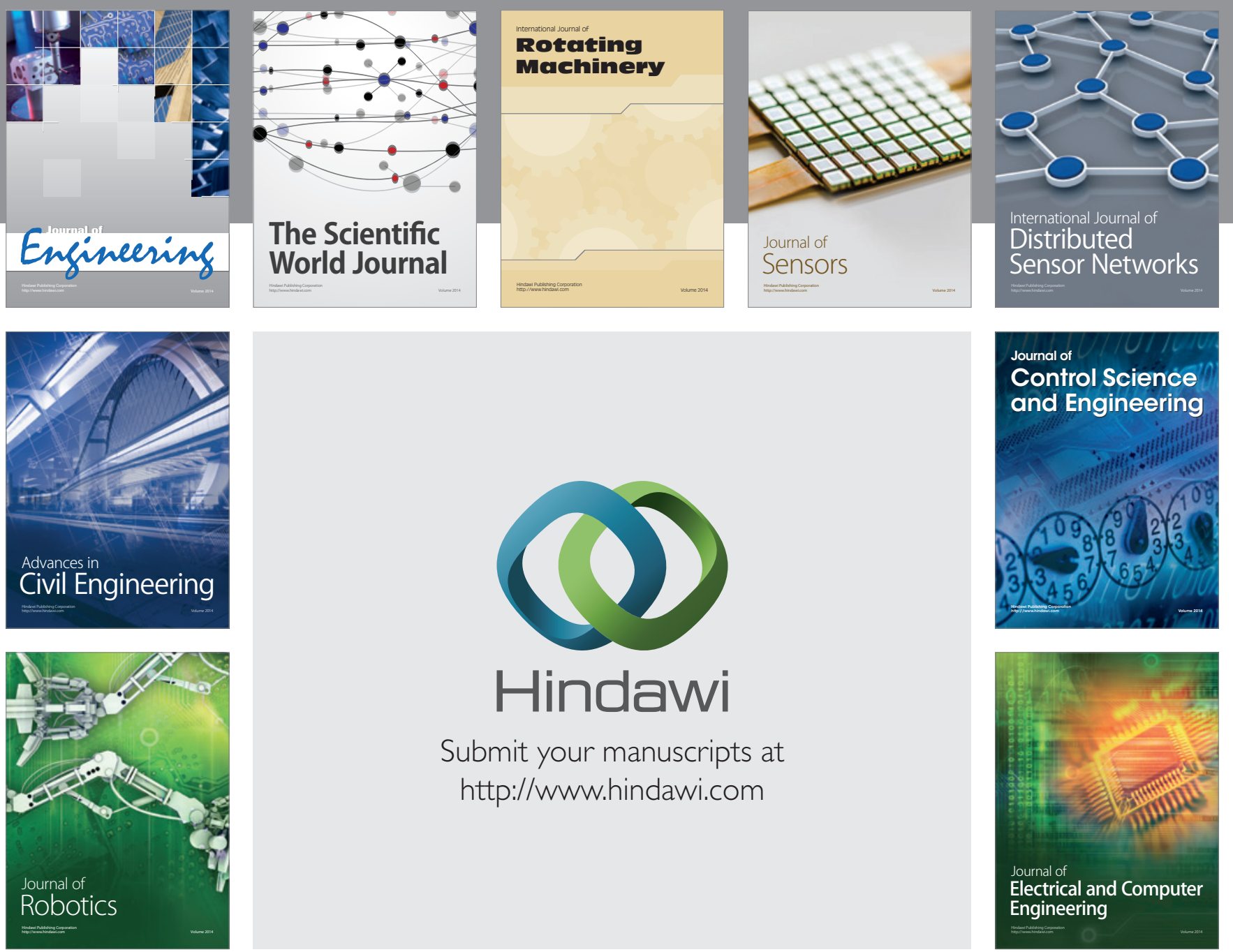

Submit your manuscripts at

http://www.hindawi.com
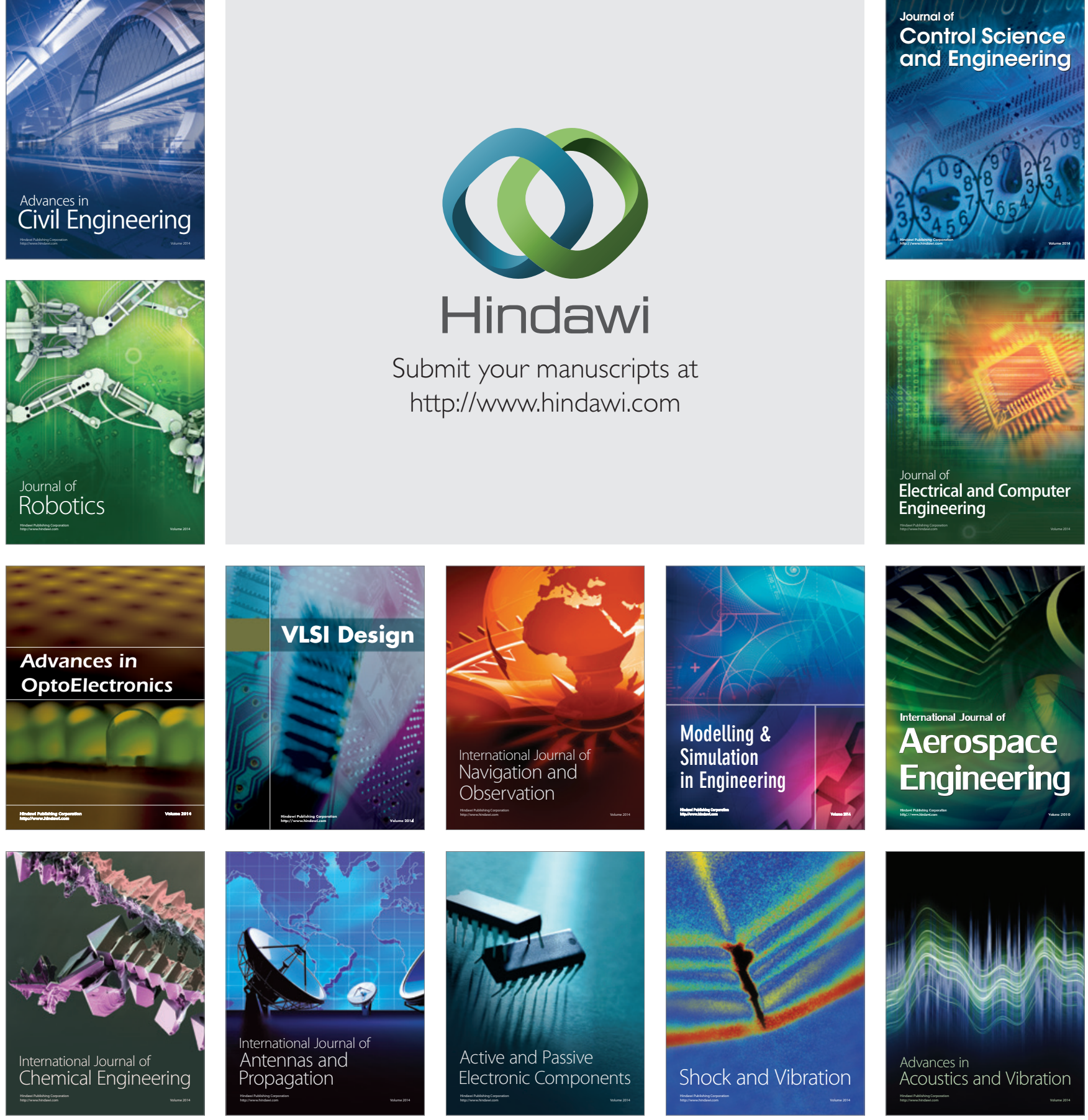